\title{
RECENT DEVELOPMENTS FOR AN IMPROVED OPERATION AT THE ESRF
}

\author{
L Hardy, JC Biasci, J. Chavanne, P. Duru, P. Elleaume, L. Farvacque, JM. Filhol, B. Joly, R. \\ Nagaoka, G. Naylor, JL Revol, A. Ropert, P. Van Vaerenbergh, U. Weinrich and L. Zhang \\ ESRF, Grenoble, France
}

\section{Abstract}

Although the ESRF has been operating smoothly for over nine years, continuous efforts are being made to improve the beam parameters whilst maintaining a high level of beam availability. The flexibility of the Machine is in permanent evolution: delivery at $5 \mathrm{GeV}$ (instead of 6 $\mathrm{GeV}$ ) was achieved for a few days, a High Focussing Optics lattice was in operation on one beam line (in order to reduce the photon beam size at the location of the sample), and emittance coupling was decreased from $1 \%$ to $0.25 \%$. All the cells have been equipped with damping links, which goal is to attenuate the vibration amplitudes of the girders. A new filling pattern, aimed at being a compromise for most of the Users, is being developed, whilst the Radio Frequency system has been completely upgraded to ensure a better redundancy and hence a better Mean Time Between Failures of this equipment. In addition to these improvements, the policy of preventive maintenance has been maintained, leading to a beam availability of $96.4 \%$ and a Mean Time Between Failures of 38 hours in year 2000

\section{THE ESRF: INTRODUCTION}

The European Synchrotron Radiation Facility (ESRF) is an X-ray source of the third generation. The accelerator complex is composed of a Linear accelerator $\left(\mathrm{e}^{-} 200\right.$ $\mathrm{MeV})$, a booster synchrotron (300 meters $-6 \mathrm{GeV})$ and a Storage Ring (844 meters).

The ESRF accelerators have been in full routine operation for over nine years. The source delivers 5500 hours of X-ray beam to nearly 40 beamlines simultaneously.

Table1: Beam parameters in the uniform filling mode

\begin{tabular}{|l|l|l|}
\hline \multicolumn{3}{|c|}{ ESRF main beam parameters } \\
\hline Particles & Electrons & \\
\hline Nominal energy & 6 & $\mathrm{GeV}$ \\
\hline Nominal intensity & 200 & $\mathrm{~mA}$ \\
\hline Associated lifetime & 82 & hours \\
\hline Horizontal emittance & $3.810^{-9}$ & $\mathrm{~m} . \mathrm{rad}$ \\
\hline Nominal coupling & $<1$ & $\%$ \\
\hline
\end{tabular}

\section{FLEXIBILITY OF BEAM DELIVERY CONDITIONS}

\subsection{Various energies...}

The nominal energy of electrons is $6 \mathrm{GeV}$. In order to increase the panel of beam delivery conditions to the users, studies were dedicated to store the beam at others energies: 4 and $5 \mathrm{GeV}$. Finally these modes were delivered for a few shifts during the Users Service Mode (USM).

- At $4 \mathrm{GeV}$, the beam delivery was done at $100 \mathrm{~mA}$. This led to a record in terms of emittance values: $\varepsilon_{\mathrm{x}}=$ $1.7 \mathrm{~nm} . \mathrm{rad}$ and $\varepsilon_{\mathrm{z}}=12 \mathrm{pm}$.rad. Although Users could make experiments up to $30 \mathrm{KeV}$, using their undulators up to the $21^{\text {st }}$ harmonics, the photon flux was found much too reduced compared to $6 \mathrm{GeV}$.

- At $5 \mathrm{GeV}$, the beam could be delivered in better conditions: beam intensity was $190 \mathrm{~mA}$ (with an associated lifetime of 17 hours). The horizontal emittance was decreased down to $2.5 \mathrm{~nm}$ rad whereas the vertical emittance could be decreased down to 7 pmrad (corresponding to a coupling of $0.25 \%$ ).

Although Users could benefit from a smaller emittance, the reduction of the photon flux for both energies was considered as too penalising by experimenters. These modes will therefore be kept in reserve for exceptional requests from Users.

\subsection{Low vertical emittance}

In routine operation, the nominal horizontal emittance of the beam is $3.810^{-9} \mathrm{~m} . \mathrm{rad}$ with an associated coupling of $0.8 \%$. One method was developed in order to further decrease the vertical emittance [ref. 1]. The principle is to use 32 skew correctors to minimise the vertical emittance measured by 2 pinhole cameras. It was possible to decrease the vertical emittance from 38 pmrad down to 10 pmrad (i.e., a coupling of $0.25 \%$ ). The beam was successfully delivered to the Users for one week in this condition (figure 1). Due to Touschek scattering, the lifetime was decreased from 65 hours to 50 hours (in $2 / 3$

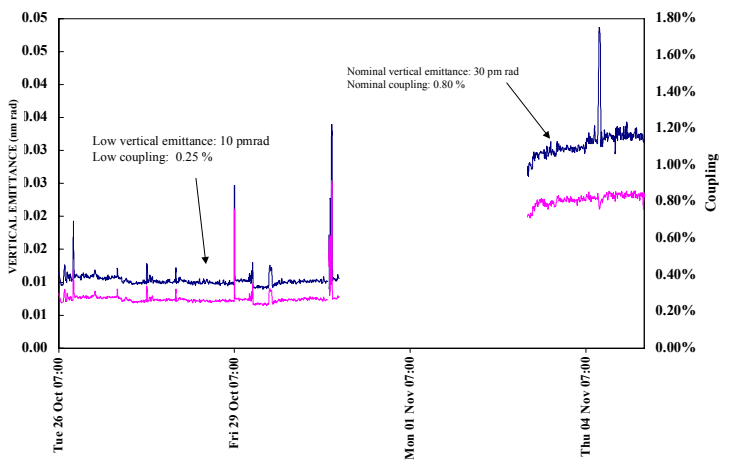

Fig.1: Beam delivery with $0.25 \%$ coupling. 
filling mode). Feedbacks from the Users were generally very positive. In particular, some of them noted a reduction of parasitic scattering in the vertical plane in the ultra-small-angle regime. The measured diffraction patterns showed a better resolution for these experiments.

\subsection{Filling modes.}

Ten different filling modes have already been developed at the ESRF. Five of them are now routinely delivered: the single bunch mode, the 16 bunch, the Hybrid mode, the $2 * 1 / 3$ filling and the uniform filling. The ideal compromise would be a mode satisfying all Users, those needing as much as possible photon flux with long lifetime and those who need a time-structured mode. It is why we develop a new hybrid mode consisting in 193 $\mathrm{mA}$ shared in $24 * 8$ bunches $+7 \mathrm{~mA}$ in a single bunch. This is expected to deliver this mode for the first time towards the end of 2001.

Until now, the single bunch mode was delivered at 15 $\mathrm{mA}$ (with a lifetime of 7 hours). Since summer 2000, it was possible to store $22 \mathrm{~mA}$ of single bunch without any instabilities. Since then, the single bunch is delivered at $20 \mathrm{~mA}$ with 7 hours lifetime and 2 coupling. Further studies to quantify this effect are on progress (ref [7]).

\subsection{Downstream Focusing Optics}

It is possible to obtain a very small X-ray size on the users sample by focusing the electron beam to a virtual point downstream the beamline. This in turn leads to a significant increase in spectral flux per unit surface. A given straight section can be tuned to a large $\beta_{\mathrm{x}}$ with nonzero $\alpha_{x}$. The new tuning implies an unsymmetrical powering of the downstream and upstream quadrupoles of the straight section concerned as well as inverted polarities on some of them. This configuration has been tested and optimised for all available filling patterns [ref 2]. It is remarkable to note that the lifetime was not affected by the breaking of the optics symetry. This option was successfully tested during normal operation mode and can be set on and off at any time.

\section{IMPROVEMENTS OF BEAM DELIVERY CONDITIONS}

\subsection{Damping links}

Through the quadrupoles, the effect of the mechanical vibrations of the magnets girders assembly (MGA) on the electron beam closed orbit is amplified more than 10 times. The main peak appears at $7 \mathrm{~Hz}$ and is mainly due to a resonance motion of the quadrupole girder in the lateral direction. Since X-ray beam stability is of prime importance for the experiments, various damping schemes to reduce these vibrations have been studied for several years. The idea is to use a sandwich structure with viscoelastic material to absorb the dynamic strain energy of the
MGA (fig.2). In addition to the existing girder supports, the damping devices are installed on the two extremities of the girder and floor. All machine girders have been recently fitted with such damping links.

The horizontal amplitude of girders vibrations have been decreased by a factor of 5 . As a consequence, the low frequencies components contained in the X-ray beam intensity spectra before the installation has now completely disappeared [ref 3,4].

Girder Mounting Fixture

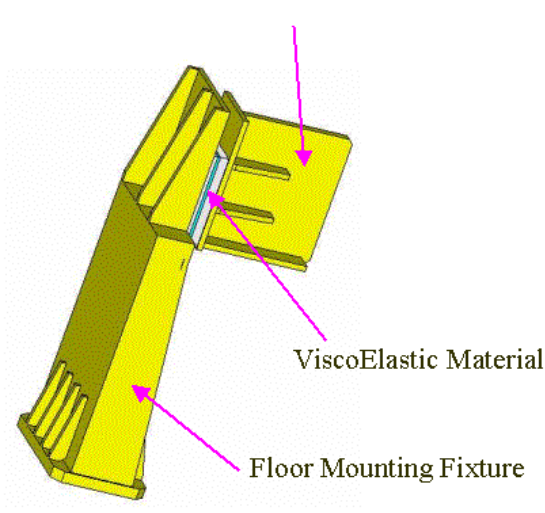

Fig.2

\subsection{A better measurement of bunch purity}

The ESRF delivers several filling modes requiring very clean bunches for the experimenters using the X-ray time structure. Until now, an avalanche photo diode was used to quantify the beam purity, by counting the photons in the parasitic bunches. This confirmed that the bunch purity was $10^{-7}$ or better. A gated high-count rate device is now used to measure the purity in the $10^{-10}$ range. It uses a beryllium window to allow the high flux of $\mathrm{Cu} \mathrm{K}$ fluorescence photons to be detected [ref.5]. However there is a great non-linearity between the counting of the main bunch and the parasitic bunches (due to the great difference in intensity). This is why the main bunch cannot be included in the gate and a calibration is needed prior to a measurement in order to establish the ratio between the main bunch and the parasitic bunches. The avalanche photodiode must also recover from the main pulse (around 10-20ns) before successive photons may be counted. This limits the use in this very high dynamic range mode to examining the purity of bunches some $20 \mathrm{~ns}$ or more after the main pulse (though this is indeed the time when the purity requirement of the users is the greatest).

\subsection{A great variety of insertion devices.}

About 60 segments of 1.6 meters each are now installed in the Storage Ring. Using a 5 meter long undulator with 34 $\mathrm{mm}$ period and a gap closed at $11 \mathrm{~mm}$, a brilliance close to $510^{20} \mathrm{phot} / \mathrm{sec} / .1 \% / \mathrm{mm}^{2} / \mathrm{mrad}^{2}$ is now reached [ref 6].

One in-vacuum undulator $(23 \mathrm{~mm}$ period / $1.6 \mathrm{~m}$ long / minimum gap of $5 \mathrm{~mm}$ ) has been in routine operation for 
1 year whereas four others are being manufactured. For a fixed energy of $12 \mathrm{keV}$ and for the same undulator's length, they produce 3 times more flux at a gap of $6 \mathrm{~mm}$ than a 'classical' undulator at $16 \mathrm{~mm}$ gap.

This summer 2001, a $3 \mathrm{~T}$ permanent magnet wiggler will be installed in place of a supra conducting wiggler. It will be dedicated to the production of circularly polarized light at high energy.

Three APPLE II undulators are now installed on the Storage Ring. They have periods of $88 \mathrm{~mm}$ and $38 \mathrm{~mm}$ and aimed to operate to a minimum gap of $16 \mathrm{~mm}$. Their goal is to produce linear/helical variable polarized X-ray beams.

\subsection{New high power Front End absorber}

Until summer 2000, the Front End movable absorber could only sustain the power generated by 2 undulators (3.2 m long) at a gap of $16 \mathrm{~mm}$. A new design of movable absorber made of Glidcop/copper has been installed in several Front ends. Using a diamond window (instead of Beryllium), they are able to sustain a power density of $400 \mathrm{~kW} / \mathrm{mrad}^{2}$. This new design allows the safe operation of a $5 \mathrm{~m}$ long undulator at a gap of $11 \mathrm{~mm}$ and at $200 \mathrm{~mA}$ intensity.

\section{THE LAST 16 MONTHS OF OPERATION}

\subsection{Some statistics}

From the $1^{\text {st }}$ January 2000 until $18^{\text {th }}$ May 2001 , the Xray source has delivered 7200 hours of beam for the Users (out of 7446 hours which were scheduled). Excellent beam availability was therefore obtained: $96.8 \%$ !

However the main figure of merit for the Users is the Mean Time Between Failures (MTBF). Once again, a record was reached during that period with a MTBF of 40 hours. The improvement of these two important figures of merit reflects a decrease of both repetitive short failures AND accidental 'long' failures.

The record time of beam delivery was reached during run 2000-03 with 360 hours (15 days) of delivery interrupted only by the Machine Dedicated Time.

\subsection{Filling modes}

The 'high intensity modes', i.e., all modes for which the beam intensity is $200 \mathrm{~mA}$ represents $70 \%$ of the delivered modes. Towards the beginning of year 2000, the uniform mode, where all the bunches are filled, was introduced. The main difficulty was to get rid of the High Order Modes (HOMs) induced in the RF cavities.

A lifetime greater than 80 hours at $200 \mathrm{~mA}$ is currently obtained in the uniform filling mode. This long lifetime and the 2 refills scheduled only twice per day insure a very stable heat load on the optics in the beamline.

\subsection{Failures and preventive maintenance}

Only 6 failures greater than 2 hours occurred from the beginning of year 2000 until May 2001. There were only 2 major failures. One was due to a broken ceramic feedthrough of an ion pump. Its replacement required to vent a vacuum vessel in order to exchange the ion pump (leading to a conditioning afterwards). The whole operation interrupted the beam for 20 hours. The second was due to a faulty device on our High Quality Power Supply (this system constituted of $10^{*} 1 \mathrm{MW}$-Diesel engines, is designed to provide the necessary power in case of a mains voltage drop or storms). Many pieces of equipment stopped and some were damaged. This interrupted the beam for 13 hours.

A failure of the synchrotron klystron may prevent to refill the Machine. In order to avoid this kind of failure, a redundant system has been recently developed. Thanks to a system of wave-guides and switches, it is now easy to power the booster synchrotron using one of the Storage Ring klystrons if necessary.

Continuing to reduce the Mean Time Between Failure (now at about 40 hours) is our first priority. This is why each beam interruption is systematically examined until it is understood and remedies are identified.

\section{REFERENCES}

[1] Work carried out at the ESRF to characterise and correct the coupling. R. Nagaoka. EPAC 2000.

[2] Horizontal focusing optics at the ESRF, A. Ropert, P. Elleaume, L. Farvacque, J. M. Hasselsweiler, EPAC2000

[3]Vibration damping systems for magnet girder assembly at the ESRF, L. Zhang, M. Lesourd, T. Lewis. PAC 2001.

[4] E-beam stability enhancement by use of damping link for magnet girder assembly at the ESRF, L. Zhang, L. Farvacque, JM Filhol and E. Plouviez. PAC 2001.

[5] A high dynamic range bunch purity tool, B. Joly and G. Naylor. DIPAC 2001.

[6] Recent achievements and future prospects of ID activities at the ESRF, Chavanne, Vanvaerenbergh, Elleaume, Guenzel. EPAC 2000.

[7] Observation, modelling and cure of transverse instabilities at the ESRF, JL Revol, R. Nagaoka, PAC 2001. 